\title{
Response to Letter to the Editor: Intragastric Balloon for Management of Severe Obesity: a Systematic Review
}

\author{
Noah Jacob Switzer ${ }^{1,2} \cdot$ Ekua Yorke $^{1} \cdot$ Xinhe Shi $^{3} \cdot$ Shahzeer Karmali $^{1,3}$
}

Published online: 28 November 2016

(C) Springer Science+Business Media New York 2016

Dear editor,

Please consider these comments in response to the Letter to the Editor submission raising concerns about our recently published article by Yorke et al., "Intragastric balloon for management of severe obesity: a systematic review [1]."

We appreciate the interest taken to our article and acknowledge the peer-reviewed critique. Our paper reflects the issue of bridging morbidly obese patients to definitive bariatric surgery with the placement of intragastric balloons (IGB). Our interest was to capture studies that reflected the use of IGB for bariatric specific weight loss only. This was reflected in our search criteria requiring both search terms for intragastric balloon and for bariatric surgery. While we understand that this narrowed focus limits the study's external validity and hence the generalizability of its findings, our question, as bariatric surgeons, was for bariatric specific weight loss.

The main concern in the Letter to the Editor was the omission of the paper by Kotzampassi et al. in our systematic review. While it indeed might be the third largest paper

Noah Jacob Switzer

nswitzer@ualberta.ca

1 Department of Surgery, University of Alberta, Edmonton, AB, Canada

2 2D, Walter C Mackenzie Health Sciences Centre, University of Alberta, 840-112 Street, Edmonton, AB T6G 2B7, Canada

3 Center for the Advancement of Minimally Invasive Surgery (CAMIS), Royal Alexandria Hospital, Edmonton, AB, Canada existing on the efficacy and safety of IGB, it simply did not meet our specific search criteria [2]. It is, however, interesting and noteworthy that the findings of our study are concordant with the endpoints in that particular study.

A few other issues were raised about methodological and citation techniques. As experienced systemic reviewers of electronic databases, we take great care in our methodologically sound principles and therefore we started our database search with the earliest year possible for PubMed citations, 1946. We can appreciate that content matter specialists might start the search upon the implementation of the IGB; however, in order to be systematic and unbiased in our search strategy, we implemented systematic and reproducible principles. The note about the inaccurate citation is valid.

We are hopeful to the future of intragastric balloons for bariatric and non-bariatric weight loss, and look forward to reading any future research in this area from the Kotzampassi et al. team.

\section{Compliance with Ethical Standards}

Conflict of Interest The authors declare that they have no conflict of interest.

\section{References}

1. Yorke E, Switzer NJ, Reso A, et al. Intragastric balloon for management of severe obesity: a systematic review. Obes Surg. 2016.

2. Kotzampassi K, Grosomanidis V, Papakostas P, et al. 500 intragastric balloons: what happens 5 years thereafter? Obes Surg. 2012;22(6): 896-903. 